\title{
Neurological features and outcome in COVID-19: dementia can predict severe disease
}

\author{
Mehran Ghaffari ${ }^{1} \cdot$ Hossein Ansari ${ }^{2,3} \cdot$ Nahid Beladimoghadam $^{1} \cdot$ Seyed Hossein Aghamiri ${ }^{1} \cdot$ Mehrdad Haghighi $^{4}$. \\ Mahmoud Nabavi ${ }^{4}$. Behnam Mansouri ${ }^{1}$. Masoud Mehrpour ${ }^{1}$. Farhad Assarzadegan ${ }^{1}$. Omid Hesami ${ }^{1}$. \\ Meghdad Sedaghat ${ }^{5} \cdot$ Mohammad Farahbakhsh $^{4} \cdot$ Behnam Safarpour Lima $^{1,6}$
}

Received: 24 August 2020 / Revised: 21 September 2020 / Accepted: 1 October 2020 / Published online: 8 January 2020

(c) Journal of NeuroVirology, Inc. 2021

\begin{abstract}
The COVID-19 pandemic has infected more than 22 million people worldwide. Although much has been learned about COVID-19, we do not know much about its neurological features and their outcome. This observational study was conducted on the patients of Imam Hossein Hospital, and 361 adult patients (214 males) with confirmed diagnosis of COVID-19 from March 5, 2020 to April 3, 2020, were enrolled. Data was gathered on age, sex, comorbidities, initial symptoms, symptoms during the disease course, neurological symptoms, and outcome. The mean age of the patients was $61.90 \pm 16.76$ years. The most common initial symptoms were cough, fever, and dyspnea. In 21 patients (5.8\%), the initial symptom was neurological. History of dementia was associated with severe COVID-19 disease (odds ratio $=1.28$ ). During the course of the disease, 186 patients $(51.52 \%)$ had at least one neurological symptom, the most common being headache (109 [30.2\%]), followed by anosmia/ageusia $(69,[19.1 \%])$, and dizziness $(54,[15 \%])$. Also, 31 patients had neurological complications $(8.58 \%)$. Anosmia, ageusia, dizziness, and headache were associated with favorable outcome $(P<0.001)$, while altered mental status and hemiparesis were associated with poor outcome. The mortality rate of patients who had neurological complications was more than twice than that of patients without neurological complication $(P=0.008)$. Almost half of the patients experienced at least one neurological symptom, which may be the initial presentation of COVID-19. Dementia appears to be associated with severe COVID-19. Mortality was higher in patients with neurological complications, and these patients needed more intensive care.
\end{abstract}

Keywords COVID-19 $\cdot$ SARS-CoV-2 $\cdot$ Neurology $\cdot$ Mortality $\cdot$ Nervous system $\cdot$ Dementia

Behnam Safarpour Lima

drbehnam_safarpour@yahoo.com; b.safarpour@sbmu.ac.ir

1 Department of Neurology, Shahid Beheshti University of Medical Sciences, Tehran, Iran

2 Headache and Facial Pain Clinic, Kaizen Brain Center, San Diego, USA

3 Department of Neurology, University of California, San Diego, USA

4 Department of Infectious Diseases, Shahid Beheshti University of Medical Sciences, Tehran, Iran

5 Department of Internal Medicine, Shahid Beheshti University of Medical Sciences, Tehran, Iran

6 Imam Hossein Medical and Educational Center, Madani Street, Tehran, Iran

\section{Introduction}

We have entered the eighth month of COVID-19 (Coronavirus disease 2019) epidemic, after it was first identified in December 2019 in Wuhan, China, and has since spread globally, and the World Health Organization declared the outbreak a public health emergency of international concern on January 30, 2020, and a pandemic on March 11. Until August 21, approximately 797,000 deaths have been occurred due to the virus, and the total number of the patients has reached more than 22 million cases throughout the world (World Health Organization 2020). COVID-19 common presentations include pyrexia, dry cough, fatigue, and shortness of breath. The severity of respiratory symptoms varies greatly and ranges from asymptomatic to pneumonia (Wu and McGoogan 2020). 
Other symptoms such as fatigue and anosmia, headache, myalgia, chills, nausea, or vomiting can also be present. Nasal congestion, diarrhea, hemoptysis, and conjunctival congestion occur less frequently (Mission W-CJ 2020). The age of the patients has been from 30 to 80 years, but we are also facing an increasing prevalence in children $(\mathrm{Wu}$ and McGoogan 2020).

The role of age, gender, association with comorbidities (hypertension, cardiovascular and cerebrovascular diseases, diabetes), smoking, body mass index, biochemical changes, and type of pulmonary involvement in chest computed tomography (CT) scan are mentioned as the major contributors to more severe disease and mortality from COVID-19 (Kakodkar et al. 2020; Williamson et al. 2020).

There are currently few reports of COVID-19 neurological features. Neurological complications of corona viruses have not been widely discussed in previous severe acute respiratory syndrome (SARS) and Middle East respiratory syndrome (MERS) epidemics. In 2004, during the SARS$\mathrm{COV}$ epidemic, nervous system was considered as a target of the virus for the first time (Ding et al. 2004).

In the present study, our primary goal was to investigate neurological features in patients with COVID-19 and their outcomes. We also evaluated the relationship between demographic characteristics of the patients, clinical manifestations, comorbidities (including previous history of neurological diseases), and laboratory findings with disease severity and mortality. It is obvious that we need more information about the factors that affect the severity and mortality of COVID-19, at present.

\section{Materials and methods}

This retrospective, observational study was conducted on the patients of Imam Hossein Hospital, a tertiary center in the east of Tehran. After the first case of the COVID-19 was reported in Iran in February 17, 2020, this hospital was assigned by government to admit and treat patients with COVID-19.

We retrospectively evaluated all the adult patients who were hospitalized with diagnosis of COVID-19 from March 5, 2020 to April 3, 2020. The diagnosis was confirmed based on two criteria: (1) patients who had a positive result on realtime reverse-transcriptase polymerase chain reaction (rRTPCR) analysis of throat swab specimens and (2) patients who had typical symptoms of the COVID-19, had a history of contact with confirmed COVID-19 patient (based on PCR) within 14 days prior to the onset of the disease, had bilateral subpleural ground glass opacities in chest CT scans, and had negative rRT-PCR results. We observed that the sensitivity of CT compared with PCR is $95.6 \%$. The documents of the patients were examined by 2 infectious disease specialists, and chest $\mathrm{CT}$ scans were re-evaluated by two trained radiologists. Patients who did not meet the above criteria were excluded from the study.

Based on the inclusion criteria mentioned above, 362 patients entered the study.

\section{Method of collecting and analyzing data}

Based on a questionnaire developed at the beginning of the study, medical records of all the patients including demographic information, history, course of the disease, doctor's instructions, nursing reports, and summary of patient discharge file were evaluated. We gathered data on age, sex, comorbidities (hypertension, cardiovascular disease, diabetes mellitus, chronic kidney disease, respiratory disease, malignancy, previous neurological disease), patient symptoms from onset to hospital admission and during the disease course (fever, cough, dyspnea, myalgia, diarrhea, nausea, vomiting, abdominal pain), central neurological symptoms (headache, dizziness, vertigo, ataxia, dysphasia, altered mental status, seizure, paresis/paralysis,), peripheral neurological symptoms (taste impairment, smell impairment, paresthesia, numbness, paresis/paralysis), and patients outcome.

Laboratory findings including complete blood count, blood biochemical analysis, C-reactive protein(CRP), creatine phosphokinase (CPK), rRT-PCR analysis of throat swab specimens (novel coronavirus 2019-nCoV nucleic acid detection kit, Sansure Biotechnology Co., Changsha, China), and findings of chest CT scans were evaluated and registered. Brain CT scan, brain magnetic resonance imaging (MRI), cerebrospinal fluid (CSF) analysis, and electrodiagnostic studies were evaluated when available and registered.

All patients were re-evaluated by phone call between 2 and 6 weeks after discharge, and any missing or inconsistent data was clarified. In the case of patients who have passed away or have not been able to provide a history, a telephone call was made to their family.

We defined the degree of severity of COVID-19 using the Diagnosis and Treatment Protocol for Novel Coronavirus Pneumonia (Trial Version 6) (Commission 2020). Mild cases consist of patients with mild symptoms and without sign of pneumonia in chest CT scan. Moderate cases had fever and respiratory symptoms with radiological findings of pneumonia. Severe cases consist of patients meeting any of the following criteria: respiratory distress (respiratory rate $\geq 30$ breaths per minute or oxygen saturation $\leq 93 \%$ at rest $)$ and/or arterial partial pressure of oxygen $\left(\mathrm{PaO}_{2}\right) /$ fraction of inspired oxygen $\left(\mathrm{FiO}_{2}\right) \leq 300 \mathrm{~mm} \mathrm{Hg}$ and/or cases with chest imaging that showed obvious lesion progression (> 50\%) within 24-48 h. Critical cases had respiratory failure or other organ failure and required mechanical ventilation or were in the shock state. We put mild and moderate cases in the "non-severe group" and severe and critical cases 
in the "severe group." Poor outcome was defined as death in the course of disease.

All neurological signs and symptoms were reviewed and confirmed by 2 trained neurologists. Major disagreement between 2 neurologists was resolved by consultation with a third reviewer.

Data were presented as mean \pm SD (standard deviation) and frequency (percentage). Independent sample $t$ test and chi-square test were used to compare normally distributed data and categorical variables between severe and nonsevere groups, respectively. Backward-selection method was performed to select the best subset of predictors. All statistical analyses were performed using SPSS, version 24 , software. The significance level was set at a 2 -sided $P$ value less than 0.05 .

\section{Results}

In this retrospective, observational study, 361 hospitalized patients with COVID-19 were evaluated including 221 patients with positive rRT-PCR results and 141 patients with clinical, epidemiologic and chest CT findings compatible with COVID-19. Among the 221 patients tested positive for rRT-PCR, 211 patients had chest CT scans with bilateral subpleural ground glass opacities. There was no statistically significant difference between the 2 groups in terms of laboratory findings (namely increased CRP and lymphopenia) and patients' outcome.

Among patients, 214 patients (59.3\%) were male and 147 (40.7\%) were female. The mean age of the patients was 61.90 years $( \pm 16.76)$. The most common initial symptoms were cough (139 [36.8\%]), fever (115 [31.9\%]), and dyspnea (95 [26.3\%]). In 21 patients (5.8\%), the initial symptom was neurological (Table 1).

During the course of the disease, the most common symptoms were dyspnea (255 [70.6\%]), cough (245 [67.9\%], fever (229 [63.4\%]), myalgia (174 [48.2\%]), and gastrointestinal symptoms (74 [30.6\%]). During the course of the disease, 186 patients $(51.52 \%)$ had at least one neurological symptom. The most common neurological symptom was headache (109 [30.2\%]), followed by anosmia/ageusia (69, $[19.1 \%])$, dizziness $(54,[15 \%])$ and altered mental status (41, [11.4\%]) (Table 2).

Hypertension was the most common comorbidity (108 [29.9\%]) followed by diabetes (99 [27.4\%] and ischemic heart disease (62 [17.2\%]) (Table 1).

With regard to the severity of the disease, 233 patients $(65.54 \%)$ were in the severe group (133 men and 100 women). The patients in the severe group were significantly older (mean [SD] age, 65.21 years [16.51] vs 55.86 years [15.56], $P<0.001)$. In the severe group, dyspnea and altered mental status were more common as initial symptoms
(30.9\% vs $18 \%, P=0.008)$ and $(7.7 \%$ vs $1.6 \%, P=0.01)$, respectively, while fever was more common in non-severe group $(43.8 \%$ vs $25.3 \%, P<0.001)$ as an initial symptom (Table 1). Statistical analysis showed that total lymphocyte count (TLC) is significantly lower, and C-reactive protein (CRP) is significantly higher in the severe group in comparison with non-severe group $(P=0.03$ and 0.003 , respectively). Hypertension and diabetes were more common in the severe group (33.9\% vs $22.7 \%, P=0.02$ and $31.3 \%$ vs $20.3 \%, P=0.02$, respectively).

With regard to outcome, the mean age of patients who died was significantly higher than patients who recovered $(70.69 \pm 15.51$ vs $56.27 \pm 14.76, P<0001)$. Also, $31.6 \%$ of patients in the severe group had a fatal outcome, while this ratio in the non-severe group was $4.7 \%(P<0.001)$. Among initial symptoms, altered mental status and dyspnea were associated with higher mortality (50\% and 39.8\% respectively, $P<0.001$ compared with other symptoms). In relation to the symptoms that appeared during the course of the disease, sore throat and gastrointestinal symptoms (diarrhea, vomiting, abdominal pain) were associated with favorable outcome $(P<0.05)$. Compared with improved patients, the mean number of lymphocytes was lower $(923.62 \pm 93.27$ vs $1234.59 \pm 49.49, P=0.02)$ and the average CRP $(80.15 \pm 7.30$ vs $57.83 \pm 3.95, P=0.01)$ and leukocyte count $(7602.78 \pm 443.78$ vs $6285.36 \pm 190.16$, $P=0.01$ ) were higher in patients with fatal outcome. We did not find statistically significant relationship between CPK level and severity of disease or patient outcome.

We evaluated the history of neurological diseases, and we found that dementia was associated with severe COVID19 disease. Using modified Poisson regression and controlling the confounding variable of age, patients with a history of dementia were 1.28 times more likely to develop severe COVID-19 disease (Table 3).

Among patients, 31 patients had neurological complications (8.58\%). The frequency of neurological complications in patients was as follows: encephalopathy without obvious metabolic or hypoxic etiology (11 patients, 3.0\%), seizures (10 patients, $2.8 \%$ ), ischemic stroke (8 patients, $2.2 \%$ ), intracerebral hemorrhage (4 patients, $1.1 \%$ ), subarachnoid hemorrhage ( 1 patient, $0.3 \%$ ), multiple sclerosis relapse (1 patient, $0.3 \%$ ), and Guillain-Barré syndrome (1 patient, $0.3 \%$ ) (Table 2).

We did not find statistically significant relationship between neurological complications and the severity of the COVID-19. In addition, no statistically significant relationship was observed between neurological complications and laboratory findings (namely increased CRP and lymphopenia).

With regard to outcome, anosmia, ageusia, dizziness, and headache were associated with more favorable outcome $(P<0.001)$, while altered mental status and hemiparesis 
Table 1 Demographic and clinical characteristics of patients with COVID-19

\begin{tabular}{|c|c|c|c|c|}
\hline Characteristics & Non-severe & Severe & Total & $P$ value \\
\hline Age (years), mean (SD) & $55.86 \pm 15.56$ & $65.21 \pm 16.51$ & $61.90 \pm 16.76$ & $<0.001$ \\
\hline \multicolumn{5}{|l|}{ Gender } \\
\hline Male & $81(63.3)$ & $133(57.1)$ & $214(59.3)$ & \\
\hline Female & $47(36.7)$ & $100(42.9)$ & $147(40.7)$ & 0.25 \\
\hline \multicolumn{5}{|l|}{ Initial symptom } \\
\hline Cough & $45(35.2)$ & $94(40.5)$ & $139(38.6)$ & 0.32 \\
\hline Fever & $56(43.8)$ & $59(25.3)$ & 115 (31.9) & $<0.001$ \\
\hline Dyspnea & $23(18)$ & $72(30.9)$ & $95(26.3)$ & 0.008 \\
\hline Myalgia & $15(11.7)$ & $24(10.3)$ & $39(10.8)$ & 0.67 \\
\hline Mental change & $2(1.6)$ & $18(7.7)$ & $20(5.5)$ & 0.01 \\
\hline Sore throat & $4(3.1)$ & $2(0.9)$ & $6(1.7)$ & 0.10 \\
\hline Seizure & $1(0.8)$ & $2(0.9)$ & $3(0.8)$ & 0.93 \\
\hline Gastrointestinal & $4(3.1)$ & $3(1.3)$ & $7(1.9)$ & 0.22 \\
\hline Hemiparesis & $4(3.1)$ & $3(1.3)$ & $7(1.9)$ & 0.22 \\
\hline Quadriparesis & $1(0.8)$ & $0(0)$ & $1(0.3)$ & 0.17 \\
\hline Aphasia & $2(1.6)$ & $1(0.4)$ & $3(0.8)$ & 0.25 \\
\hline Headache & $5(3.9)$ & $2(0.9)$ & $7(1.9)$ & 0.04 \\
\hline \multicolumn{5}{|l|}{ Comorbidities } \\
\hline Diabetes & $26(20.3)$ & $73(31.3)$ & $99(27.4)$ & 0.02 \\
\hline Hypertension & $29(22.7)$ & $79(33.9)$ & 108 (29.9) & 0.02 \\
\hline Ischemic heart disease & $18(14.1)$ & $44(18.9)$ & $62(17.2)$ & 0.24 \\
\hline Respiratory disease & $6(4.7)$ & $11(4.7)$ & $17(4.7)$ & 0.98 \\
\hline Malignancy & $5(3.9)$ & $11(4.7)$ & $16(4.4)$ & 0.72 \\
\hline Chronic kidney disease & $4(3.1)$ & $14(6)$ & $18(5)$ & 0.22 \\
\hline Hypothyroidism & $4(3.1)$ & $8(3.4)$ & $12(3.3)$ & 0.87 \\
\hline \multicolumn{5}{|c|}{ History of neurological disease } \\
\hline Migraine & $3(2.3)$ & $3(1.3)$ & $6(1.7)$ & 0.47 \\
\hline Myelopathy & $1(0.8)$ & $0(0)$ & $1(0.3)$ & 0.17 \\
\hline Epilepsy & $4(3.1)$ & $8(3.4)$ & $12(3.3)$ & 0.87 \\
\hline Dementia & $0(0)$ & $8(3.4)$ & $8(2.2)$ & 0.03 \\
\hline Stroke & $3(2.3)$ & $11(4.7)$ & $14(3.9)$ & 0.25 \\
\hline Multiple sclerosis & $1(0.8)$ & $0(0)$ & $1(0.3)$ & 0.17 \\
\hline Parkinson disease & $1(0.8)$ & $5(2.1)$ & $6(1.7)$ & 0.35 \\
\hline \multicolumn{5}{|c|}{ Symptoms during the disease course } \\
\hline Fever & $86(67.2)$ & $143(61.4)$ & $229(63.4)$ & 0.27 \\
\hline Cough & $87(68)$ & $158(67.8)$ & $245(67.9)$ & 0.97 \\
\hline Dyspnea & $70(54.7)$ & $185(79.4)$ & $255(70.6)$ & $<0.001$ \\
\hline Myalgia & $73(57)$ & $101(43.3)$ & $174(48.2)$ & 0.01 \\
\hline Sore throat & $12(9.4)$ & $20(8.6)$ & $32(8.9)$ & 0.80 \\
\hline \multicolumn{5}{|l|}{ Gastrointestinal } \\
\hline Diarrhea & $31(24.2)$ & $29(12.4)$ & $60(16.6)$ & 0.004 \\
\hline Vomiting & $24(18.7)$ & $43(18.4)$ & $67(18.5)$ & 0.94 \\
\hline Abdominal pain & $5(3.9)$ & $2(0.8)$ & $7(1.9)$ & 0.03 \\
\hline
\end{tabular}

were associated with less favorable outcome. Also, $62.5 \%$ of patients with a history of dementia and $42.9 \%$ of patients with a history of stroke had a fatal outcome $(P=0.04)$. Neurological complications were significantly associated with increased mortality. The mortality rate of patients with COVID-19 who had neurological complications was more than twice that of patients without neurological complication $(P=0.008)$. 
Table 2 Neurological features in patients with COVID-19

\begin{tabular}{lllll}
\hline Characteristics & $\begin{array}{l}\text { Non-severe } \\
\text { Number }(\%)\end{array}$ & Severe & Total & $P$ value \\
\hline $\begin{array}{l}\text { Neurological symptom } \\
\text { Headache }\end{array}$ & $49(38.3)$ & $60(25.8)$ & $109(30.2)$ & 0.01 \\
Dizziness & $12(9.4)$ & $22(9.4)$ & $34(9.4)$ & 0.98 \\
Vertigo & $25(19.5)$ & $29(12.4)$ & $54(15)$ & 0.07 \\
Paresis & $6(4.7)$ & $6(2.6)$ & $12(3.3)$ & 0.28 \\
Anosmia & $27(21.8)$ & $42(24.4)$ & $69(19.1)$ & 0.59 \\
Ageusia & $28(21.9)$ & $41(17.6)$ & $69(19.1)$ & 0.32 \\
Sensory & $6(4.7)$ & $7(3)$ & $13(3.6)$ & 0.41 \\
Hearing loss & $2(1.6)$ & $1(0.4)$ & $3(0.8)$ & 0.25 \\
Dysphasia & $4(3.1)$ & $9(3.9)$ & $13(3.6)$ & 0.72 \\
Ataxia & $9(7)$ & $11(4.7)$ & $20(5.5)$ & 0.36 \\
Altered mental status & $10(7.8)$ & $31(13.3)$ & $41(11.4)$ & 0.11 \\
Neurological disorder & & & & \\
Ischemic stroke & $3(2.3)$ & $5(2.1)$ & $8(2.2)$ & 0.90 \\
Intracerebral hemorrhage & $1(0.8)$ & $3(1.3)$ & $4(1.1)$ & 0.66 \\
Subarachnoid hemorrhage & $0(0)$ & $1(0.4)$ & $1(0.3)$ & 0.45 \\
Encephalopathy & $2(1.6)$ & $9(3.9)$ & $11(3)$ & 0.22 \\
Encephalitis & $\mathrm{NA}$ & $\mathrm{NA}$ & $\mathrm{NA}$ & $\mathrm{NA}$ \\
Seizure & $3(2.3)$ & $7(3)$ & $10(2.8)$ & 0.71 \\
\hline
\end{tabular}

$N A$ not available

Table 3 Age adjusted relative risk for dementia obtained from modified Poisson regression

\begin{tabular}{llll}
\hline Variables & $\begin{array}{l}\text { Relative risk for } \\
\text { dementia }\end{array}$ & $95 \%$ CI & $P$ value \\
\hline Dementia & 1.28 & $1.15-1.42$ & $<0.001$ \\
Age & 1.01 & $1.00-1.02$ & $<0.001$ \\
\hline
\end{tabular}

CI confidence interval

\section{Discussion}

Our result showed that in 21 patients $(5.8 \%)$, the initial symptom of COVID-19 was neurological. We also observed that history of dementia was associated with severe COVID-19 disease. During the course of the disease, 186 patients $(51.52 \%)$ had at least one neurological symptom, and 31 patients had neurological complications $(8.58 \%)$. It was found that the mortality rate of patients who had neurological complications was more than twice than that of patients without neurological complication.

Coronaviruses are a large group of viruses that are capable of causing a variety of diseases, from the respiratory tract to the gastrointestinal tract infections in mammals and birds. Coronaviruses cause about $15 \%$ of human common colds in winter and spring. In most cases, the infection is mild and self-limited but occasionally, severe acute respiratory infections occur. Coronaviruses usually have a specific host, but sometimes, due to mutation or recombination, they cross the species barrier and transfer from one species to another (Mandell et al. 2020). In the last 20 years, three species of animal coronaviruses have caused epidemics in humans. The SARS epidemic occurred between 2002 and 2004. In 2012, the MERS epidemic began and continues, and since 2019, COVID-19 or SARS CoV-2 has caused epidemic and pandemic across the globe and with capability of survival in the surfaces and transmission with respiratory droplets and the presence of asymptomatic carriers; it has affected most of the world's human societies. It also seems that neurological manifestations (NeuroCOVID) are part of the COVID-19 clinical picture (Leonardi et al. 2020).

Currently, COVID-19's main diagnostic method is rRTPCR, but its sensitivity is about $71 \%$ (Fang et al. 2020); thus, a negative test result does not necessarily rule out the disease. These negatives could result from improper sampling techniques, low viral load in the area sampled, or mutations in the viral genome (Udugama et al. 2020). Instead, chest CT has a high sensitivity for diagnosis of COVID-19 (97\%), but its accuracy is $68 \%$ and specificity is $25 \%$ (Ai et al. 2019). In the study conducted by Jian-Long He et al., initial RT-PCR and chest $\mathrm{CT}$ had comparable diagnostic power in the detection of suspected COVID-19 patients outside the epidemic center (Wuhan, China) and concluded that to compensate 
for potential risk of false-negative PCR, chest CT should be performed for clinically suspected patients with negative initial RT-PCR result (He et al. 2020). Previous studies and recommendations from China CDC (Commission 2020) considered the same management for confirmed COVID19 cases (with positive PCR) and suspected cases (with negative PCR). As we are in the middle of the COVID-19 pandemic, we considered the patients with typical COVID19 symptoms, who had a history of contact with confirmed COVID-19 patient within previous 14 days, had bilateral subpleural ground glass opacities in chest CT scans, and had negative rRT-PCR results, as COVID-19 patients and included them in the study. In our study, the sensitivity of chest CT was $95.6 \%$ and there was no statistically significant difference between the 2 groups in terms of laboratory findings and patients' outcome.

In the present study, in line with the findings of Mirsoleymani et al., Huang et al., and Richardson et al., the majority of patients $(59.3 \%)$ were male and most common comorbidities were hypertension and diabetes (Huang et al. 2020; Mirsoleymani 2020; Richardson et al. 2020).

Several studies have evaluated the relationship between demographic characteristics, comorbidities, and laboratory findings with disease severity. In this study, as well, in the severe group, the patients were older (Gong et al. 2020; Huang et al. 2020), diabetes and hypertension were more common (Mirsoleymani 2020), lymphocyte count was significantly lower (Gong et al. 2020; Wan et al. 2020), and CRP was significantly higher (Gao et al. 2020; Gong et al. 2020; Huang et al. 2020; Ling 2020; Wan et al. 2020) in comparison with non-severe group. We also found that, when considering the initial symptom at the disease onset, dyspnea and altered mental status were associated with severe disease. Other studies have also shown that more severe infection may accompany altered mental status and cerebrovascular disease with higher probabilities (Mao et al. 2020b).

With regard to outcome, in line with study of Huang et al., older age and lymphopenia were associated with increased mortality (Huang et al. 2020). We also found that increased CRP, and altered mental status and dyspnea at the disease onset, was associated with high mortality, while sore throat and gastrointestinal symptoms were associated with favorable prognosis. The classification of patients into severe and non-severe can be prognostically valuable; $31.6 \%$ of patients in the severe group had a fatal outcome, while this rate in the non-severe group was $4.7 \%$.

In a prospective study, by Mao et al., $36.4 \%$ of patients with COVID-19 had neurological manifestations (Mao et al. 2020a). In the present study, consistent with the findings of Mao et al., neurological manifestations were common, but the frequency of neurological symptoms was higher $(51.52 \%)$, which can be related to how information is collected. When data is gathered only through electronic medical records, some information, especially mild symptoms (e.g., anosmia, ageusia, headache, dizziness), may not be obtained, while telephone follow up may reveal them. In a study by Wan et al., $17.4 \%$ of patients had headache (Wan et al. 2020), while in our study, $30.2 \%$ of patients complained of headache, which may be due to different ways of data collection, mentioned above.

In our study, dementia was associated with severe COVID-19 disease. Further studies, and the study of more patients with a history of dementia who have been diagnosed with COVID-19, can clarify this issue. Concerning dementia, a combination of proposed pathophysiology may play a role. However, considering the neurotropic potential of COVID-19 (Baig et al. 2020), and the well-described role of neuroinflammation in dementia (Chen et al. 2015; Ozben and Ozben 2019), it is possible that patients with more severe diseases are prone to develop dementia.

In the present study, contrary to the conclusion by Mao et al. (2020a), we did not find statistically significant relationship between neurological disorders and neither the severity of the COVID-19 nor with laboratory findings (namely, lymphopenia and increased CRP). It is yet to be elucidated that the virus invades the brain directly; the nervous system is harmed through a systemic inflammatory response or virus-induced immunosuppression. There are few published articles on neurological manifestations of coronaviruses in previous MERS-COV and SARS-COV epidemics. In 2004, possibility of CNS infection by SARS coronavirus in mice was raised (Lau et al. 2004). A report of virus isolation from the brain of a patient was published in 2005 (Xu et al. 2005). In 2016, it was shown that SARS$\mathrm{COV}$ and MERS-COV viruses have reached the brainstem in a study in mice (Li et al. 2016). A decade ago, the role of angiotensin converting enzyme 2 (ACE2) receptors as a target for SARS-CoV and the location of these receptors on vascular endothelium were mentioned in animal studies (Lau and Peiris 2005; Poyiadji et al. 2020). Studies have reported some pathologic changes in the central nervous system after COVID-19, such as hemorrhagic white matter, axonal injury, leukocyte infiltration, and ADEM-like appearance (Tabary et al. 2020). Many mechanisms have been proposed to justify NeuroCOVID. The presence of ACE-2 receptor in the brain, cytokine overproduction and toxicity, and vasculotropic and neurotropic nature of virus are among these proposed mechanisms (Aghagoli et al. 2020).

Similar to SARS-CoV, the SARS-CoV-2 virus exploits the ACE2 receptor to enter the cell. The brain expresses ACE2 receptors, which have been detected over glial cells and neurons, making them a potential target of COVID-19 (Baig et al. 2020).

The virus path to the nervous system is not yet clear. Studies show that the virus spreads to the nervous system 
via hematogenous or lymphatics routes. Animal studies have revealed the initial invasion of corona viruses into the peripheral nerves and then the trans-synaptic transfer and access to the central nervous system ( $\mathrm{Li}$ et al. 2012). Due to the occurrence of olfactory disturbance in the early stages of the disease, retrograde invasion through cribriform plate of the ethmoid bone adjacent to the olfactory bulb has been proposed too (Liu et al. 2019). Other potential route of brain and CNS involvement could be hypoxia and immune injury.

At present study, 21 patients (5.8\%) presented with neurological symptoms and did not show typical COVID-19 symptoms before admission. We do not know if this is a coincidence or if there is a cause-and-effect relationship, but this finding suggests that during the pandemic, every physician, who visits a patient with neurological symptom, should keep in mind the possibility of COVID-19. Our study population represents a snapshot of hospitalized patients with neurological complications associated with COVID19. Larger, ideally prospective, studies should identify the broader cohort of COVID-19 patients both in and outside hospitals, with capture-recapture analysis and health record linkage to determine clearer estimates of the prevalence of these complications. Studies suggest performing continuing documentation of neurological symptoms and signs, timely CSF testing, EEG, and brain autopsies of COVID-19 patients to boost current understanding of the neurological alterations of COVID-19 (Gklinos 2020).

\section{Conclusion}

Almost half of our patients experienced at least one neurological symptom during the course of the disease, and in $5.8 \%$ of the patients, the initial presentation of COVID-19 was neurologic. Neurological disorders were significantly associated with increased mortality. The mortality rate of patients with COVID-19 who had neurological complications was more than twice that of patients without neurological complication, which shows that these patients need more intensive care. Among neurological diseases, dementia appears to be associated with severe COVID-19. Mortality is much higher in patients with neurological complications, and these patients need more intensive care.

Acknowledgments We would like to express our great appreciation to Dr Hussein Soleimantabar for his valuable and constructive suggestions during the planning and development of this research work. We also appreciate the help of Clinical Research Development Unit, Imam Hossein Hospital, Shahid Beheshti University of Medical Sciences. We also thank the patients and their families for their excellent cooperation in providing information for this research.

Data availability Individual participant data that underlie the results reported in this article will be shared, after de-identification, immediately following publication, with no end date, to anyone who wishes to access the data for any purpose. Proposals should be directed to b.safarpour@sbmu.ac.ir; to gain access, data requestors will need to sign a data access agreement.

\section{Compliance with ethical standards}

Ethical approval Before data gathering, verbal consents were taken from patients or relatives of patients who were not able to give consent. All of the information obtained from the medical records, and additional information from the patient follow-up, remained completely confidential. This observational study was confirmed by the ethics committee of Shahid Beheshti University of Medical Sciences, Tehran, Iran, on March 20.

\section{References}

World Health Organization, official site (2020) Coronavirus disease 2019. Available at: 22 May 2020, 04:30

Aghagoli G, Gallo Marin B, Katchur NJ, Chaves-Sell F, Asaad WF, Murphy SA (2020) Neurological involvement in COVID-19 and potential mechanisms: a review. Neurocrit Care 1-10

Ai T, Yang Z, Hou H, Zhan C, Chen C, Lv W, Tao Q, Sun Z, Xia L (2019) Correlation of chest CT and RT-PCR testing in coronavirus disease (COVID-19) in China: a report of 1014 cases [published online ahead of print February 26, 2020]. Radiology 10

Baig AM, Khaleeq A, Ali U, Syeda H (2020) Evidence of the COVID19 virus targeting the CNS: tissue distribution, host-virus interaction, and proposed neurotropic mechanisms. ACS chemical neuroscience 11:995-998

Chen Y, Brennan-Minnella AM, Sheth S, El-Benna J, Swanson RA (2015) Tat-NR2B9c prevents excitotoxic neuronal superoxide production. J Cereb Blood Flow Metab 35:739-742

Commission NH (2020) Diagnosis and treatment protocol for novel coronavirus peumonia (trial version 7). Chinese Med J 133:E027-E027

Ding Y, He L, Zhang Q, Huang Z, Che X, Hou J, Wang H, Shen H, Qiu L, Li Z (2004) Organ distribution of severe acute respiratory syndrome (SARS) associated coronavirus (SARS-CoV) in SARS patients: implications for pathogenesis and virus transmission pathways. The Journal of Pathology: A Journal of the Pathological Society of Great Britain and Ireland 203:622-630

Fang Y, Zhang H, Xie J, Lin M, Ying L, Pang P, Ji W (2020) Sensitivity of chest CT for COVID-19: comparison to RT-PCR. Radiology 200432

Gao Y, Li T, Han M, Li X, Wu D, Xu Y, Zhu Y, Liu Y, Wang X, Wang L (2020) Diagnostic utility of clinical laboratory data determinations for patients with the severe COVID-19. J Med Virol

Gklinos P (2020) Neurological manifestations of COVID-19: a review of what we know so far. J Neurology 1

Gong J, Dong H, Xia SQ, Huang YZ, Wang D, Zhao Y, Liu W, Tu S, Zhang M, Wang Q (2020) Correlation analysis between disease severity and inflammation-related parameters in patients with COVID-19 pneumonia. MedRxiv

He J-L, Luo L, Luo Z-D, Lyu J-X, Ng M-Y, Shen X-P, Wen Z (2020) Diagnostic performance between CT and initial real-time RT-PCR for clinically suspected 2019 coronavirus disease (COVID-19) patients outside Wuhan, China. Resp Med 105980

Huang C, Wang Y, Li X, Ren L, Zhao J, Hu Y, Zhang L, Fan G, Xu J, $\mathrm{Gu} X$ (2020) Clinical features of patients infected with 2019 novel coronavirus in Wuhan, China. The lancet 395:497-506 
Kakodkar P, Kaka N, Baig M (2020) A comprehensive literature review on the clinical presentation, and management of the pandemic coronavirus disease 2019 (COVID-19). Cureus 12

Lau K-K, Yu W-C, Chu C-M, Lau S-T, Sheng B, Yuen K-Y (2004) Possible central nervous system infection by SARS coronavirus. Emerg Infect Dis 10:342

Lau YL, Peiris JM (2005) Pathogenesis of severe acute respiratory syndrome. Curr Opin Immunol 17:404-410

Leonardi M, Padovani A, McArthur JC (2020) Neurological manifestations associated with COVID-19: a review and a call for action. J Neurol 267:1573-1576

Li K, Wohlford-Lenane C, Perlman S, Zhao J, Jewell AK, Reznikov LR, Gibson-Corley KN, Meyerholz DK, McCray PB Jr (2016) Middle East respiratory syndrome coronavirus causes multiple organ damage and lethal disease in mice transgenic for human dipeptidyl peptidase 4. J Infect Dis 213:712-722

Li Y-C, Bai W-Z, Hirano N, Hayashida T, Hashikawa T (2012) Coronavirus infection of rat dorsal root ganglia: ultrastructural characterization of viral replication, transfer, and the early response of satellite cells. Virus Res 163:628-635

Ling W (2020) C-reactive protein levels in the early stage of COVID19. Medecine et maladies infectieuses

Liu P, Chen W, Chen J-P (2019) Viral metagenomics revealed Sendai virus and coronavirus infection of Malayan Pangolins (Manis javanica). Viruses 11:979

Mandell G, Dolin R, Bennett J (2020) Mandell, Douglas, and Bennett's principles and practice of infectious diseases. Elsevier

Mao L, Jin H, Wang M, Hu Y, Chen S, He Q, Chang J, Hong C, Zhou Y, Wang D (2020) Neurologic manifestations of hospitalized patients with coronavirus disease 2019 in Wuhan China. JAMA neurology 77:683-690

Mao L, Wang M, Chen S, He Q, Chang J, Hong C, Zhou Y, Wang D, Miao X, Hu Y (2020b) Neurological manifestations of hospitalized patients with COVID-19 in Wuhan, China: a retrospective case series study

Mirsoleymani S (2020) Risk factors for severe coronavirus disease 2019 (COVID-19) among Iranian patients: who was more vulnerable?

Mission W-CJ (2020) Report of the WHO-China Joint Mission on Coronavirus Disease 2019 (COVID-19), Geneva
Ozben T, Ozben S (2019) Neuro-inflammation and anti-inflammatory treatment options for Alzheimer's disease. Clin Biochem 72:87-89

Poyiadji N, Shahin G, Noujaim D, Stone M, Patel S, Griffith B (2020) COVID-19-associated acute hemorrhagic necrotizing encephalopathy: CT and MRI features. Radiology 201187

Richardson S, Hirsch J, Narasimhan M, Crawford J, McGinn T, Davidson K, Consortium NC-R (2020) Barnaby DP. Becker LB, Chelico JD, Cohen SL, Cookingham J, Coppa K, Diefenbach MA, Dominello AJ, Duer-Hefele J, Falzon L, Gitlin J, Hajizadeh N, Harvin TG, Hirschwerk DA, Kim EJ, Kozel ZM, Marrast LM, Mogavero JN, Osorio GA, Qiu M, Zanos TP Presenting characteristics, comorbidities, and outcomes among 5700

Tabary M, Khanmohammadi S, Araghi F, Dadkhahfar S, Tavangar SM (2020) Pathologic features of COVID-19: a concise review. Pathology Res Prac 153097

Udugama B, Kadhiresan P, Kozlowski HN, Malekjahani A, Osborne M, Li VY, Chen H, Mubareka S, Gubbay JB, Chan WC (2020) Diagnosing COVID-19: the disease and tools for detection. ACS Nano 14:3822-3835

Wan S, Xiang Y, Fang W, Zheng Y, Li B, Hu Y, Lang C, Huang D, Sun Q, Xiong Y (2020) Clinical features and treatment of COVID-19 patients in northeast Chongqing. J Med Virology

Williamson E, Walker AJ, Bhaskaran KJ, Bacon S, Bates C, Morton CE, Curtis HJ, Mehrkar A, Evans D, Inglesby P (2020) OpenSAFELY: factors associated with COVID-19-related hospital death in the linked electronic health records of 17 million adult NHS patients. medRxiv

Wu Z, McGoogan JM (2020) Characteristics of and important lessons from the coronavirus disease 2019 (COVID-19) outbreak in China: summary of a report of 72314 cases from the Chinese Center for Disease Control and Prevention. JAMA 323:1239-1242

Xu J, Zhong S, Liu J, Li L, Li Y, Wu X, Li Z, Deng P, Zhang J, Zhong $\mathrm{N}$ (2005) Detection of severe acute respiratory syndrome coronavirus in the brain: potential role of the chemokine mig in pathogenesis. Clin Infect Dis 41:1089-1096

Publisher's Note Springer Nature remains neutral with regard to jurisdictional claims in published maps and institutional affiliations. 